\title{
THE MEAN-VALUE ITERATION FOR SET-VALUED MAPPINGS
}

\author{
PETER K. F. KUHFITTIG
}

ABSTRACT. In this note Krasnoselskii's iteration procedure

$$
x_{n+1}=\frac{1}{2}(I+T) x_{n}
$$

is extended to certain classes of set-valued mappings.

1. Introduction. Let $C$ be a convex subset of a Banach space $B$ and $T$ a self-mapping of $C$ and consider the following iteration process of a type introduced by Mann [7]: for an arbitrary starting point $x_{0} \in C$

$$
x_{n+1}=\left(1-c_{n}\right) x_{n}+c_{n} T x_{n}, \quad n=0,1,2, \ldots,
$$

where $c_{n} \in[a, b]$ for $0<a<b<1$. The special case $c_{n}=\frac{1}{2}$ for all $n$ was first introduced by Krasnoselskii [5], who showed that the sequence converges to a fixed point of $T$ if $T$ is nonexpansive, $B$ uniformly convex, and $C$ compact. This result remains valid if $c_{n}=\alpha, 0<\alpha<1$ (Schaefer [12]). Moreover, it is sufficient to assume that $B$ is strictly convex (Edelstein [3]). Retaining uniform convexity, Browder and Petryshyn [1] assumed $C$ to be closed and $T$ demicompact. Under the latter conditions, the sequence (*) converges to a fixed point of $T$ if $T$ is merely continuous and quasinonexpansive, that is, nonexpansive about its set of fixed points, assumed nonempty. (See Corollary which follows.) The iteration (*) has been investigated by Senter and Dotson [13].

In this paper we shall consider an analogous iteration for a mapping $T$ : $C \rightarrow K(C)$, where $K(C)$ is the family of nonempty compact subsets of $C$. It is assumed that one fixed point $z$ is known and that $T$ is nonexpansive about this point, that is, for all $x \in C$

$$
D(T x, T z)<\|x-z\|,
$$

where $D$ is the Hausdorff metric on $K(C)$. The iteration procedure is designed to generate additional fixed points.

Regarding the existence of fixed points, it was shown by Lim [6] that if $C$ is a convex closed and bounded subset of a uniformly convex Banach space, then a nonexpansive mapping $T: C \rightarrow K(C)$ has a fixed point. This result has recently been extended by Downing and Kirk [2].

2. The sequence. Let $z \in T z$ be the known fixed point. Since $T x$ is compact and $D$ the Hausdorff metric, we can find for every $x \in C$ a point $p_{x} \in T x$ such that

$$
\left\|z-p_{x}\right\|<D(T z, T x) \text {. }
$$

Received by the editors September 5, 1979 and, in revised form, December 3, 1979.

AMS (MOS) subject classifications (1970). Primary 47H10; Secondary 54C60.

Key words and phrases. Fixed points, set-valued mappings, iteration. 
Using this information, suppose we construct a sequence $\left\{x_{n}\right\}$ in $C$ as follows: let $x_{0} \in C$ and $p_{0} \in T x_{0}$. Next let

$$
x_{1}=\left(1-c_{0}\right) x_{0}+c_{0} p_{0}
$$

where $c_{0} \in[a, b], 0<a<b<1$. Then we can find $p_{1} \in T x_{1}$ such that

$$
\left\|z-p_{1}\right\|<D\left(T z, T x_{1}\right)
$$

by the prior comments. Now let

$$
x_{2}=\left(1-c_{1}\right) x_{1}+c_{1} p_{1} .
$$

Since $T x_{2}$ is compact, we can find $p_{2} \in T x_{2}$ such that

$$
\left\|z-p_{2}\right\|<D\left(T z, T x_{2}\right) \text {. }
$$

Continuing in this manner

$$
x_{n+1}=\left(1-c_{n}\right) x_{n}+c_{n} p_{n}, \quad n=0,1,2, \ldots,
$$

where $c_{n} \in[a, b]$ for $0<a<b<1, p_{n} \in T x_{n}$, and

$$
\left\|z-p_{n}\right\|<D\left(T z, T x_{n}\right) \text {. }
$$

Since $T$ is not even assumed to be quasinonexpansive, we do require continuity in the following sense.

Definition 1. A mapping $T: C \rightarrow K(C)$ is continuous if for any sequence $\left\{y_{n}\right\}$ in $C, y_{n} \rightarrow y$ implies that $T y_{n} \rightarrow T y$.

Definition 2 (Petryshyn [10]). A mapping $U: C \rightarrow B$ of a subset $C$ of a Banach space $B$ into $B$ is said to be demicompact if whenever $\left\{x_{n}\right\} \subset C$ is a bounded sequence and $\left\{x_{n}-U x_{n}\right\}$ is a convergent sequence, then there exists a subsequence $\left\{x_{n_{i}}\right\}$ which is convergent.

For set-valued mappings we have the following analogous definition.

Definition 3. A mapping $T: C \rightarrow K(C)$ will be called demicompact if whenever $\left\{x_{n}\right\} \subset C$ is a bounded sequence and $\left\{\operatorname{dist}\left(x_{n}, T x_{n}\right)\right\}$ is a convergent sequence, then there is a subsequence $\left\{x_{n_{i}}\right\}$ which is convergent.

In the proof of the first theorem we are going to need the following two lemmas.

LEMMA 1 (SchAefER [12]). Let $B$ be a uniformly convex Banach space. Then for $\varepsilon>0, d>0$, and $\alpha \in(0,1)$ the inequalities $\|x\|<d,\|y\|<d$, and $\|x-y\|>\varepsilon$ imply that

$$
\|(1-\alpha) x+\alpha y\|<[1-2 \delta(\varepsilon / d) \min (1-\alpha, \alpha)] d
$$

$\delta$ is strictly increasing.

LEMMA 2 (NADLER [9]). Let $\left\{A_{n}\right\}$ be a sequence of sets in $K(C)$ and suppose $\lim _{n \rightarrow \infty} D\left(A_{n}, A_{0}\right)=0$, where $A_{0} \in K(C)$. Then if $x_{n} \in A_{n}, n=1,2, \ldots$, and if $\lim _{n \rightarrow \infty} x_{n}=x_{0}$, it follows that $x_{0} \in A_{0}$.

\section{Results.}

THEOREM 1. Let $C$ be a nonempty convex closed subset of a uniformly convex Banach space $B$. If $T: C \rightarrow K(C)$ is a continuous demicompact mapping which is nonexpansive about a known fixed point $z$, then for the sequence $\left\{x_{n}\right\}$ defined previously, (a) there exists a subsequence $\left\{x_{n_{i}}\right\}$ converging to a fixed point of $T$ and 
(b) every cluster point of $\left\{x_{n}\right\}$ is a fixed point of T. (In particular, every convergent subsequence of $\left\{x_{n}\right\}$ converges to a fixed point.)

Proof. The first step is to show that for the sequence $\left\{x_{n}\right\}$ constructed previously

$$
\left\|x_{n}-p_{n}\right\| \rightarrow 0 \text { as } n \rightarrow \infty .
$$

If not, then there exists a subsequence $\left\{x_{n}\right\}$ of $\left\{x_{n}\right\}$ and a number $\varepsilon>0$ such that

$$
\left\|x_{n_{j}}-p_{n_{j}}\right\|>\varepsilon \text {. }
$$

Since $p_{n} \in T x_{n}$,

$$
\left\|z-p_{n}\right\|<D\left(T z, T x_{n}\right)<\left\|z-x_{n}\right\| .
$$

Then by (1), (2) and Lemma 1 there exists

$$
\delta=\delta\left(\varepsilon /\left\|z-x_{n_{j}}\right\|\right)>0
$$

such that

$$
\begin{aligned}
\left\|z-x_{n_{j+1}}\right\| & =\left\|z-\left(1-c_{n_{j}}\right) x_{n_{j}}-c_{n_{j}} p_{n_{j}}\right\| \\
& =\left\|\left(1-c_{n_{j}}\right)\left(z-x_{n_{j}}\right)+c_{n_{j}}\left(z-p_{n_{j}}\right)\right\| \\
& \leqslant(1-\delta \gamma)\left\|z-x_{n_{j}}\right\|,
\end{aligned}
$$

where $\gamma=2 \min \left(1-c_{n_{j}}, c_{n_{j}}\right)$. From

$$
\left\|z-x_{n+1}\right\|=\left\|\left(1-c_{n}\right)\left(z-x_{n}\right)+c_{n}\left(z-p_{n}\right)\right\|<\left\|z-x_{n}\right\|,
$$

the sequence $\left\{\left\|z-x_{n}\right\|\right\}$ is nonincreasing, and since $\delta$ is strictly increasing, the sequence

$$
\left\{\delta\left(\varepsilon /\left\|z-x_{n}\right\|\right)\right\}
$$

in nondecreasing. Since we also have

$$
\left\|z-x_{n j}\right\|<\left\|z-x_{n_{j-1}+1}\right\|<(1-\delta \gamma)\left\|z-x_{n_{j-1}}\right\|
$$

for

$$
\delta=\delta\left(\varepsilon /\left\|z-x_{n_{j-1}}\right\|\right)
$$

and

$$
\gamma=2 \min \left(1-c_{n_{j-1}}, c_{n_{j-1}}\right)
$$

it follows that

$$
\left\|z-x_{n}\right\| \rightarrow 0 \text { as } j \rightarrow \infty \text {. }
$$

By (2) $\left\|z-p_{n_{j}}\right\| \rightarrow 0$, whence $\left\|x_{n_{j}}-p_{n_{j}}\right\| \rightarrow 0$ as $j \rightarrow \infty$, contradicting statement (1). Hence

$$
\left\|x_{n}-p_{n}\right\| \rightarrow 0 \text { as } n \rightarrow \infty
$$

which was to be shown.

It now follows from (4) that $\operatorname{dist}\left(x_{n}, T x_{n}\right) \rightarrow 0$ as $n \rightarrow \infty$. Moreover, by (3), $\left\{x_{n}\right\}$ is a bounded sequence. So by demicompactness there exists a subsequence $\left\{x_{n_{i}}\right\}$ of 
$\left\{x_{n}\right\}$ such that $x_{n_{i}} \rightarrow z_{0} \in C$. Also, from

$$
\left\|p_{n_{i}}-z_{0}\right\|<\left\|p_{n_{i}}-x_{n_{i}}\right\|+\left\|x_{n_{i}}-z_{0}\right\|
$$

we have that $p_{n_{i}} \rightarrow z_{0}$. But $T x_{n_{i}} \rightarrow T z_{0}$ by continuity. Consequently, since $p_{n_{i}} \in T x_{n_{i}}$, $z_{0} \in T z_{0}$ by Lemma 2 .

Finally, if $w_{0}$ is a cluster point of $\left\{x_{n}\right\}$, there exists a subsequence converging to $w_{0}$, which is a fixed point by the above argument. This completes the proof.

Recall from $\$ 2$ that $p_{x} \in T x$ is a point for which $\left\|z-p_{x}\right\|<D(T z, T x)$. Suppose for every such $p_{x} \in T x$ and $p_{y} \in T y, T: C \rightarrow K(C)$ satisfies the condition

$$
D(T x, T y)<\alpha\left\|x-p_{x}\right\|+\beta\left\|y-p_{y}\right\|
$$

for all $x, y \in C$ and $\alpha, \beta \in[0, \infty)$.

Then if $\alpha=\beta \in\left[0, \frac{1}{2}\right]$ and if $T$ is a point-to-point mapping, $T$ is a Kannan mapping, first introduced by Kannan [4]. Such mappings have been studied extensively.

If $p_{w}$ is chosen (without reference to the fixed point $z$ ) so that $\left\|w-p_{w}\right\|=$ $\operatorname{dist}(w, T w)$ and if $\alpha=\beta=\frac{1}{2}$, then $T$ becomes a set-valued Kannan mapping. Such mappings were studied by Shiau, et al. [14], [15]. Clearly, any Kannan mapping is of Type A.

Theorem 2. If $T$ in Theorem 1 is of Type A, then $T x_{n} \rightarrow T z_{0}$, where $z_{0} \in T z_{0}$.

Proof. For every $\varepsilon_{0}>0$ there exists $N>0$ such that

$$
D\left(T x_{n}, T x_{m}\right)<\alpha\left\|x_{n}-p_{n}\right\|+\beta\left\|x_{m}-p_{m}\right\|<\varepsilon
$$

for all $m, n>N$, so that $\left\{T x_{n}\right\}$ is a Cauchy sequence. Since $C$ is complete, $(K(C), D)$ is complete (Michael [8]). Hence $T x_{n} \rightarrow L \in K(C)$. Since $x_{n_{n}} \rightarrow z_{0}$, $T x_{n_{i}} \rightarrow T z_{0}$, so that $L=T z_{0}$.

The result of Theorem 2 is, in one sense, the best possible. For if $T$ is a nonexpansive set-valued mapping, then the natural analogue of Krasnoselskii's procedure is the following: let $x_{0} \in C, q_{0} \in T x_{0}$, and $x_{1}=\frac{1}{2} x_{0}+\frac{1}{2} q_{0}$. Now choose $q_{1} \in T x_{1}$ such that

$$
\left\|q_{1}-q_{0}\right\|<D\left(T x_{1}, T x_{0}\right)
$$

In general, $x_{n+1}=\frac{1}{2} x_{n}+\frac{1}{2} q_{n}$, where $q_{n} \in T x_{n}$ and

$$
\left\|q_{n}-q_{n-1}\right\|<D\left(T x_{n}, T x_{n-1}\right)
$$

whence

$$
\left\|q_{n}-q_{n-1}\right\|<\left\|x_{n}-x_{n-1}\right\| \text {. }
$$

This construction fails, however, as can be seen from the mapping $T: R \rightarrow K(R)$ defined by $T x=[x-1, x+1]$. If for $T x_{n}=\left[x_{n}-1, x_{n}+1\right]$, we choose $q_{n}=x_{n}$ +1 , then the resulting sequence has no convergent subsequence.

If $T$ is a point-to-point mapping, then $q_{n}=T x_{n}$, and $\left\{x_{n}\right\}$ reduces to Krasnoselskii's iteration. Now suppose $T$ is continuous, demicompact, and quasinonexpansive with a nonempty set $F$ of fixed points. Returning to the sequence (*), if $z \in F$, then

$$
\left\|z-T x_{n}\right\|<\left\|z-x_{n}\right\|
$$


and, by the proof of Theorem 1, there exists a subsequence $\left\{x_{n_{n}}\right\}$ for which $x_{n_{i}} \rightarrow z_{0}=T z_{0}$. Since $\left\{\left\|z_{0}-x_{n}\right\|\right\}$ is clearly nonincreasing, $x_{n} \rightarrow z_{0}$. This proves the following

COROllaRy. Let $C$ be a nonempty convex closed subset of a uniformly convex Banach space. If $T: C \rightarrow C$ is a continuous demicompact quasinonexpansive mapping with a nonempty set of fixed points, then the sequence (*) converges to a fixed point of $T$.

This result is similar to Theorem $1.1^{\prime}$ in [11] and Theorem 2 in [13].

\section{REFERENCES}

1. F. E. Browder and W. V. Petryshyn, The solution by iteration of nonlinear functional equations in Banach spaces, Bull. Amer. Math. Soc. 72 (1966), 571-575.

2. D. Downing and W. A. Kirk, Fixed point theorems for set-valued mappings in metric and Banach spaces, Math. Japon. 22 (1977), 99-112.

3. M. Edelstein, A remark on a theorem of M. A. Krasnoselskii, Amer. Math. Monthly 73 (1966), 509-510.

4. R. Kannan, Some results on fixed points. II, Amer. Math. Monthly 76 (1969), 405-408.

5. M. A. Krasnoselskii, Two observations about the method of successive approximations, Uspehi Mat. Nauk 10 (1955), 123-127. (Russian)

6. T. C. Lim, A fixed point theorem for multivalued nonexpansive mappings in a uniformly convex Banach space, Bull. Amer. Math. Soc. 80 (1974), 1123-1126.

7. W. R. Mann, Mean value methods in iterations, Proc. Amer. Math. Soc. 4 (1953), 506-510.

8. E. Michael, Topologies of spaces of subsets, Trans. Amer. Math. Soc. 71 (1951), 151-182.

9. S. B. Nadler, Jr., Multi-valued contraction mappings, Pacific J. Math. 30 (1969), 475-488.

10. W. V. Petryshyn, Construction of fixed points of demicompact mappings in Hilbert spaces, J. Math. Anal. Appl. 14 (1966), 274-284.

11. W. V. Petryshyn and T. E. Williamson, Strong and weak convengence of the sequence of successive approximations of quasi-nonexpansive mappings, J. Math. Anal. Appl. 43 (1973), 459-497.

12. H. Schaefer, Ueber die Methode sukzessiver Approximationen, Jber. Deutsch. Math.-Verein. 59 (1957), 131-140.

13. H. F. Senter and W. G. Dotson, Jr., Approximating fixed points of nonexpansive mappings, Proc. Amer. Math. Soc. 44 (1974), 375-380.

14. C. Shiau, K.-K. Tan and C. S. Wong, Quasi-nonexpansive multi-valued maps and selections, Fund. Math. 87 (1975), 109-119.

15. __ A class of quasi-nonexpansive multi-valued maps, Canad. Math. Bull. 18 (1975), 709-714.

Department of Mathematics, Milwaukge School of Engineering, Milwaukge, Wisconsin 53201 